\title{
Protection and Practice of Overheating of Electrical Contact of Power Transmission and Transformation Equipment
}

\author{
Wang Guogang* \\ Beijing Guodian Futong Science and \\ Technology Development Co., Ltd \\ Beijing, China \\ e-mail: ssnabi@126.com
}

\section{Li Guangpeng}

Shijiazhuang Tiedao University

Country Beijing Guodian Futong Science and

Technology Development Co., Ltd

Beijing, China

e-mail: guangpengli@sina.cn

\author{
Wu Liusuo \\ Beijing Guodian Futong Science and \\ Technology Development Co., Ltd \\ Beijing, China \\ e-mail: wuliusuo@sgepri.sgcc.com.cn
}

\author{
Zhang Guanglei \\ Shijiazhuang Tiedao University \\ Shijiazhuang, China \\ e-mail: zhgl@stdu.edu.cn
}

\begin{abstract}
Electrical power connector is generally a weak link in the power system, which is probably the most frequent source of detriment to the overall performance of a power system. In order to solve overheating of connector, authors surveyed many substations in service and analyzed the deterioration mechanism. We found that the main overheating spots have been concentrated on clamps, busbus joint, isolation switch, etc. Based on service condition and ambient environment of equipment, connector overheating was investigated from aspects of corrosion, fretting, great loads, etc. Coated with electrical joint compound is one of control techniques. The performance of electrical joint compound was examined by testing, and its long-term service condition was tracked. The electrical joint compound can effectively resolve the overheating defects in electrical contact, because it not only decreases the contact resistance and the temperature-rise degree, but also improves the corrosion resistance. By comparing the success of using qualified electrical joint compound with the failure of using inferior electrical joint compound, electrical joint compound must be carefully selected and subject to strict inspection before putting into service.
\end{abstract}

Keywords-contactor; overheating; corrosion; great loads; electrical joint compound

\section{INTRODUCTION}

Electrical contact of power transmission and transformation equipment is an integral part of the power network. The closed electric contact has to perform its function of passing electric current reliably and with no change in its contact resistance for the duration of its life. The contacts have to perform this function even though the ambient atmosphere in which they reside contains pollutants which can cause the contacts to tarnish or corrode. However, with the steadily increasing in electricity consumption and more complex using conditions, the contacts have to carry greater loads and operate at more complex environments than in the past. Overheating of electrical contact is thus a very common phenomenon in the electric power industry. It almost occurs everywhere in power transmission and transformation equipment. Overheating of electrical contact may bring a lot of troubles to power industry, which cannot only cause overheating burns, power outages, but also be a waste of energy. Accidents caused by electrical contact are remarkably increasing ${ }^{[1]}$. There are many connector overheating accidents: 83 overheating defects were found in the inspection process for a super high voltage electric network in $2011^{[2]}$; An overheating accident was happened in bus connector in a substation.

There are main reasons for these accidents: first, there is a general lack of awareness of the problem, since connection deterioration is a time-related progress; second, the specific features of connection deterioration are not readily recognizable, because the identification of the degradation mechanism is difficult; in addition, mounting evidence shows that the standards currently used by manufacturers to qualify connectors do not satisfactorily reflect their ability to perform under varied and adverse field conditions.

Above all, analysis for the reason of the overheating of electrical contact and timely processing are necessary for the safe operation and reliable power supply. The authors analyzed and discussed the overheating phenomenon through field research, theoretical analysis, field application and post-tracking its results.

\section{OVERHEATING SPOTS}

The overheating spots of power transmission and transformation equipment were probed by infrared diagnostic techniques ${ }^{[3]}$ in Shanxi Jincheng Substations. The test did large area detection on service equipment used by infrared cameras. The results showed that the 
overheating spots were mainly found on the joints of equipment. For example, the contact surfaces of clamps (Fig.1(a)), isolation switch (Fig .1(b)). Fig .1 shows photo of the overheating spots.

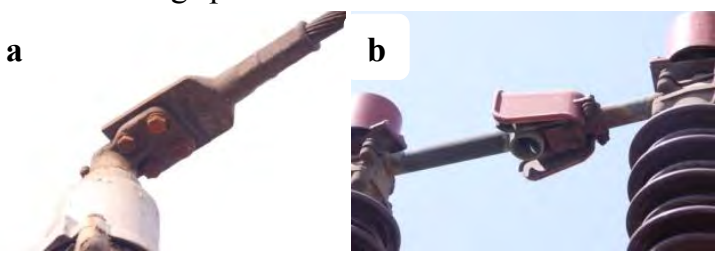

Figure 1. Photo of overheating spots

According to incomplete statistics, about 45 percent of the wiring clamps have a heating phenomenon. From "Fig .1" we can see that different degrees of corrosion were found on contact surface. Such corrosion caused the difficulty of parts replacement. Even sometimes it needs to replace the entire device, which bring a great deal of inconvenience to the maintenance and cause great economic losses. Corrosion is one of the main factors that cause the overheating of device. With the voltage level rising and transport current increasing, this heating trend was intensified under the corrosive effect. As a result, contact surface shaped welding morphology or even burned.

\section{OVERHEATING THEORY}

From the above, we found that overheating spots are usually accompanied by varying degrees of corrosion. Corrosion is one of the incentives cause overheating of connectors, but not the only factor. The influence factors of overheating of electrical contact are diverse, such as material properties, climate and environment and operating conditions. These factors can be divided into the following categories.

\section{Subjective Factors}

There are many human factors cause abnormal heat in electrical contacts: contact surface not properly handled; gap between the connectors is large, which is easy access to corrosive media; connection interface pressure is not enough; connector lacks the necessary protection.

\section{Objective Factors}

The actual contact area is not enough

All solid surfaces are rough on the micro-scale. Surface micro-roughness consists of peaks and valleys whose shape, height variations, average separation and other geometrical characteristics depend on fine details of the surface generation process ${ }^{[4]}$, as shown in Fig .2 .

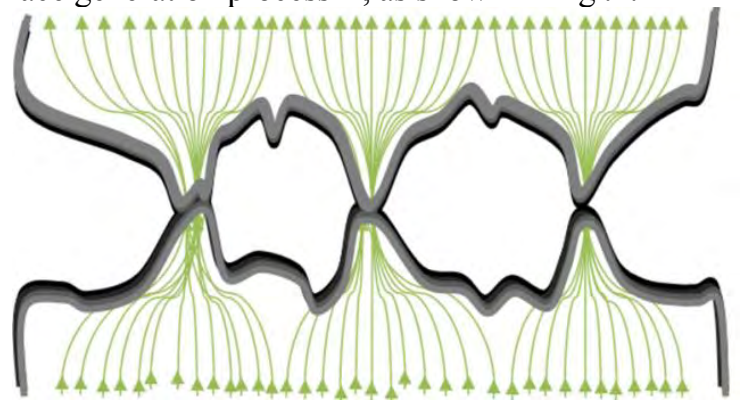

Figure 2. A schematic view of metal contact points
The mating components are metals in a bulk electrical interface. They shall be humped a location contact when put two metals together. A resistance is then generated since the current is restricted. Furthermore, the contacting surfaces are often covered with oxide or other electrically insulative layers. Generally, the interface becomes electrically conductive only when mental-to-mental contact spots are produced, where electrically insulative films are ruptured or displaced at contacting surface asperities. The area of electrical contact is thus appreciably smaller than the area of true mechanical contact. The total interfacial resistance provided by the constriction and film resistances determines the contact resistance of the interface, which cause the heating phenomenon.

\section{Contact Tarnishing And Corrosion}

\section{Electrical Injury}

Electrical injury is also called as electro-corrosion, which is caused by the migration of a large amount of surface electrons. When current is high $\left(>104 \mathrm{~A} / \mathrm{cm}^{2}\right)$, atomic vibration distance dramatically increase because of the effects of electron scattering. In heating effect of high field the migration of a large amount of atoms causes the corrosion of materials ${ }^{[5]}$. Runde ${ }^{[6]}$ found that electron migration was the essential reason for the deterioration of electrical contact materials.

\section{Galvanic Corrosion}

In electric network, the most common connection is copper-aluminum connection. In the presence of corrosive medium, the galvanic corrosion will occur because of the potential difference between them. Galvanic corrosion is very common for the electrical contact. Actually, the chemical corrosion and galvanic corrosion are acted synergistically and dependent on the conditions, such as the moist and electrolyte.

\section{Oxidation Corrosion}

The electrical connection materials are aluminum alloys, copper alloys, silvering or galvanizing copper alloys. It is well known that aluminum is easily oxidized; copper is easily corroded by halogen, sulfur, selenium; silver is sensitive to sulfide.

Lin \& Zhang ${ }^{[7]}$ reported that dust in air can corrode and deteriorate electrical contact and even seriously damage electrical contact. Chen Wei ${ }^{[8]}$ explained the mechanisms and dynamics principle of electrical contact corrosion in detail.

\section{Fretting Damage}

Fretting damage is defined as surface deterioration caused by fast and slight vibration, which can be resulted from mechanical vibration, the difference of thermal expansion coefficient and stress relaxation. Micro vibration will not only induce wear, corrosion, but also fatigue of materials. The mechanisms are quite complex ${ }^{[9]}$.

High load operation

Steadily increasing energy consumption in densely populated areas imposes severe operating conditions on transmission and distribution systems, which have to carry greater loads than in the past. Despite the use of various preventive-maintenance measures, there are still a number of overheating areas that can adversely affect system reliability ${ }^{[10]}$. 


\section{Management Of Overheating Defects}

Above all, in order to solve the problem of overheating of electrical contact, we not only need to effectively control human factors, but also limit objective factors. People consider a variety of control techniques. Electrical joint compound coated on contact surface is one of the many power complex protective measures. According to IEEE Std 524-2003 ${ }^{[11]}$ and the national standard GB 50389-2006 [12] and other standard specification, the electrical connections are required to be coated with electrical joint compound or similar functional materials. Such materials are widely abroad applications and run better ${ }^{[13]}$. At present, we thank the electrical joint compound, which is one of the most effective solutions to solve the electrical contact overheating. The liquid for electrical joint compound is silicone oil, and then zinc powder as conducting material is mixed into silicone oil. Other anticorrosion additive and antioxidant additive are also added into silicone oil. The electrical joint compound has good electrical conductivity. It can remarkably decrease contact resistance and temperature-rise rate, and has good resistance to corrosion [14-16]

\section{Contact Resistance}

The contact resistance of aluminum clamps was tested in Shanxi Jincheng Wozhuang Substation. Aluminum clamps were chosen according to the standard and its contact area is $100 \mathrm{~mm} \times 100 \mathrm{~mm}$. Field testing picture is shown as Fig.3.

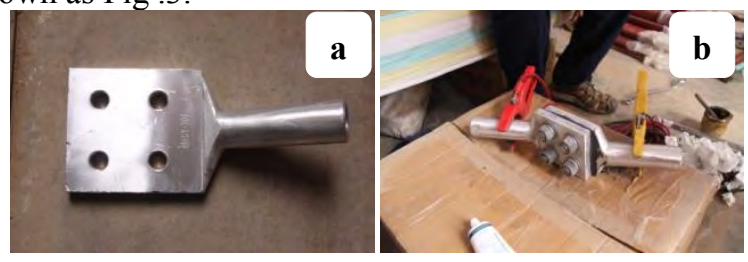

Figure 3. Sample photo for contact resistance experiments

The experiment was done in two steps. First, we test contact resistance of aluminum clamps without anything (Fig .3(a)); then, contact resistance of aluminum clamps coated electrical joint compound was test (Fig .3(b)). The testing results are shown as TABLE I

TABLE I

\begin{tabular}{ccc}
\hline samples & $\begin{array}{c}\text { without anything } \\
\mathbf{R} 1(\boldsymbol{\mu} \boldsymbol{\Omega})\end{array}$ & $\begin{array}{c}\text { coated electrical } \\
\text { joint compound R2 } \\
(\boldsymbol{\mu} \boldsymbol{\Omega})\end{array}$ \\
\hline A & 12.6 & 7.1 \\
B & 12.8 & 7.0 \\
C & 13.1 & 6.9 \\
\hline
\end{tabular}

TABLE I shows that electrical joint compound can effectively reduce the contact resistance.

\section{Temperature}

In Shanxi Jincheng Taitou Substation, temperature of contact was tested on the joints of the capacitor, as illustrated in Fig .4.

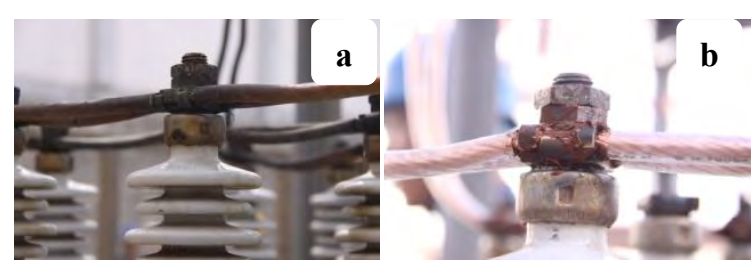

Figure 4. Before and after coating

The experiment is divided into two steps. First, the temperature of joints was tested without any treatment (Fig .4(a)). Then the temperature of joints was tested after cleaned and coated by electrical joint compound (Fig .4(b)). This test was carried out on four points of contact. The result is shown as TABLE II

TABLE II

\begin{tabular}{ccc}
\hline Contact points & $\begin{array}{c}\text { Temperature before } \\
\text { processing }\left({ }^{\circ} \mathrm{C}\right)\end{array}$ & $\begin{array}{c}\text { Temperature } \\
\text { after } \\
\text { processing }\left({ }^{\circ} \mathrm{C}\right)\end{array}$ \\
\hline $\mathrm{A}$ & $\geqq 100$ & $\leqq 45$ \\
$\mathrm{~B}$ & $\geqq 100$ & $\leqq 45$ \\
$\mathrm{C}$ & $\geqq 100$ & $\leqq 45$ \\
$\mathrm{D}$ & $\geqq 100$ & $\leqq 45$ \\
\hline
\end{tabular}

The temperature of joint after processing is far lower than that before processing. It means electrical joint compound can effectively decrease the temperature of electrical contact and reduce the overheating defects.

\section{Successful and Failure Applications}

\section{Successful Applications}

Electrical joint compound was used on wire connectors, power distribution cabinets, reactors and other parts in Beijing Fangshan Electricity Transmission and Distribution Company on October 2009. Since then, the company never has abnormal heat generation, its equipment runs well. Electrical joint compound effectively solves the overheating problems of capacitor joints as well as bus. Electrical joint compound was also tested and applied to $\pm 800 \mathrm{kV}$ high voltage reactor, instead of traditional copper plating process or tin plating process in Beijing Electric Power Equipment Factory, which not only solve the joints corrosion problem, but also effectively solve the joints overheating problem.

\section{Failure Applications}

Electrical contacts occured discharge ablation failure when isolation switch long-term in a standby spare put into operation in a substation in Shandong Province ${ }^{[17]}$.The reason is the poor quality of electricity joint compound. In the natural environment it has been severely dusty and dry, and difficult to clean up (Fig .5(a)), resulting in the isolation switch dynamic and static contact is not effective contact, causing overheating, contact pillow melting, burned accidents (Fig .5(b)). Burned photo was shown as Fig . $5^{[17]}$

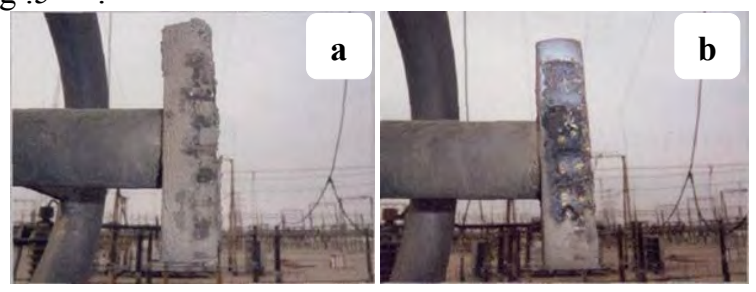

Figure 5. Ablative marks on the contact 


\section{DISCUSSION}

Through the above tests and application cases we can find that electrical joint compound can effectively solve the electrical connection overheating. There are so many reasons for that: Electrical joint compound can fill the gap between two metal contact surfaces, which increases the actual contact area; there are conducting particles in electrical joint compound which can pass power effectively because of its "contact effect" and "tunneling effect"; the electrical joint compound protects the electrical contact from corrosion because corrosive medium, such as air, moisture, is blocked outside the gap; the possibility of wear of connector reduces since the electrical joint compound has lubricating effect.

However, there are electrical contact burned accidents even the contact surface is coated with electrical joint compound. The reasons are as follows.

\section{Coating inferior electrical joint compound}

With the application of electrical joint compound, domestic manufacturers have sprung up. Electrical joint compound is widely used in various fields of electrical industry. However, due to the spotty performance, the effect of applicable is very different. Many manufacturers simply pursue interests and ignore product qualities. The product often occurs ages flatten, oil precipitation, harden and heating loss phenomenon.

\section{Non-standard operation}

Due to the lack of operating specifications, the high technological requirements and the complex operation, making electrical joint compound did not play the desired effect. For instance, electrical joint compound coating thickness is uneven, too thin, or too thick; because of the presence of glitches, pitting, oil and oxide film the contact surface is not flat, which needs a lot of work to be cleaned; the contact force is hard to control, which directly affect the quality of contact properties.

\section{CONCLUSION}

To avoid electrical connection overheating incidents, that need regular checks on common hot spots, analyze the cause of overheating and timely processing. Infrared detection technology is one of effective means of detection, but due to complex environmental conditions, it is necessary to adopt a comprehensive analysis of a variety of methods;

The use of electrical joint compound on electrical contacts protects contact surfaces from corrosion, reduces friction and wear and thereby reduces the contact resistance. Accordingly, electrical joint compound is an effective mean to solve the temperature rise, which not only can greatly enhance the reliability of electricity, but also play a role in energy conservation to reduce wire losses.

The choice of electrical joint compound cannot be arbitery. Electrical joint compound must be chosen according to the actual application conditions, rigorously tested and operated by corresponding standards, making sure the validity of electrical joint compound.

\section{ACKNOWLEDGMENT}

The financial support of this work extended by the Science and Technology Project of State Grid Shanxi Electric Power Company (Grant 5205E01351DG) and the Science and Technology Project of State Grid Corporation (Grant FTZX201404) are gratefully acknowledged.

\section{REFERENCES}

[1] Zhang Ji-gao. A Summary Report on the Mechanism of Electric Contact Failure Due to Particle Contamination [C]. Electrical Contacts. 2011: 1-8

[2] Zhu Shan-jun, Zhang Jian, Mi Yong. Analysis and Prevention Measures of Overheating of Outdoor Isolation Switch fever [J] Shanxi Electric Power, 2012, (04): 22-25.

[3] China Electricity Council. DL/T 664-1999. Application rules of infrared diagnosis for live electrical equipment [S]. Beijing: 2008.

[4] TR Thomas. Rough Surfaces [M], New York: Longman, 1982.

[5] Tu King-ning. Solder Joint Technology [M]. 2010.

[6] Runde M, Hodne E, Totdal B. Current-induced aging of contact spots[C]. Electrical Contacts. 1989: 213-220.

[7] Lin Xue-yan, Zhang Ji-gao. Dust corrosion [C]. Electrical Contacts. 2004: 255-262.

[8] W. Chen, Y. Zhen. Review of the mechanism of electric contact material corrosion [J]. Electric Materials, 2010, vol. 3: 3-8.

[9] Braunovic M. Fretting Damage in Tin-plated Aluminum and Copper Connectors [J]. Components, Hybrids and Manufacturing Technology, 1989, vol. 12: 215-223.

[10] Paul G. Slade. Electrical Contacts: Principles and Applications [M], 2 ed. CRC Press, 2013.

[11] Transmission \& Distribution Committee, IEEE std 524-2003. IEEE guide to the installation of overhead transmission line conductor [S]. NY: The Institute of Electrical and Electronics Engineers, inc. 2004

[12] China Electricity Council. GB 50389-2006. Code for construction and acceptance of $750 \mathrm{kv}$ overhead transmission line [S]. Beijing: China Building Industry Press, 2006.

[13] Bella H Chudnovsky. Lubrication of electrical contacts [C]. Electrical Contacts. 2005: 107-114.

[14] Li Xing-wei, Wang Gou-gang, Qiang Chun-mei. Experimental study on the influence of electrical joint compound on anticorrosion property of electrical contact [J]. Power Construction, 2011. 32(8): 99-102.

[15] Xiong Xin-xin. The proper use of "conductive paste" during electrical equipment maintenance [J]. Science and Technology Information, 2009(21): 323.

[16] Gong Bing-lin. The conductive paste used in the electrical connection [J]. Public Electricity, 2009(6): 32.

[17] Liu Dong-hai. Analysis of $220 \mathrm{kV}$ isolation switch failure [J] China Power Enterprise Management, 2011, 135-137. 